Pacific Journal of Mathematics

TOPOLOGICAL IDENTIFICATION OF MULTIPLE SOLUTIONS 


\title{
TOPOLOGICAL IDENTIFICATION \\ OF MULTIPLE SOLUTIONS \\ TO PARAMETRIZED NONLINEAR EQUATIONS
}

\author{
ROBERT F. BROWN \\ Dedicated to the memory of James Dugundji
}

Let $L: E \rightarrow F$ be an isomorphism of Banach spaces, let $H: E \times \mathbf{R}^{n}$ $\rightarrow F$ be a completely continuous mapping, and let $B: E \rightarrow \mathbf{R}^{n}$ be a bounded linear mapping onto a euclidean space. The solutions $(y, \lambda)$ to the problem

$$
\left\{\begin{array}{l}
L y=H(y, \lambda), \\
B y=0
\end{array}\right.
$$

can be represented as the fixed points of a mapping $T: E \times \mathbf{R}^{n} \rightarrow E \times$ $\mathbf{R}^{n}$. Neilsen fixed point theory may be extended to produce lower bounds for the number of fixed points of such maps. Problems of the type $(*)$ include boundary value problems for ordinary differential systems of the form:

$$
\left\{\begin{array}{l}
y^{\prime \prime}=h\left(x, y, y^{\prime}, \lambda\right), \\
y(0)=y(1)=0,
\end{array}\right.
$$

where $y=y(x):[0,1] \rightarrow \mathbf{R}^{n}$ and $\lambda \in \mathbf{R}^{n}$, satisfying an additional condition such as $y(1 / 2)=0$ or $\int_{0}^{1} y(t) d t=A$ for a given $A \in \mathbf{R}^{n}$.

A standard technique in nonlinear analysis, for establishing that an equation has a solution, consists of finding a mapping whose fixed points correspond to the solutions of the equation and then applying a topological fixed point theorem. A fixed point theory initiated by Jakob Nielsen in the 1920s is concerned with the number (rather than just the existence) of fixed points [8]. In 1950, Jean Leray [11] suggested that Nielsen fixed point theory might therefore be useful in proving that equations have multiple solutions. In this paper, we apply Neilsen fixed point theory to a class of parametrized equations in which the parameter space is finite-dimensional.

Section 1 is concerned with the modification of some techniques from [1], to obtain a form of Nielsen theory suited to the analytic problem. Section 2 presents a description of the problem in operator theoretic language and contains the main result of the paper (Theorem 2.3) which gives sufficient conditions for the topological methods of the first section 
to be applicable to this problem. Some specfic types of problems for parametrized nonlinear differential systems which can be formulated as operator theory problems of the type of $\S 2$ are described in $\S 3$. In $\$ 4$ we examine more closely one of the problem types of $\S 3$ : the three-point boundary value problem for a second-order system of parametrized nonlinear ordinary differential equations. The final section presents the analysis of two specific problems which illustrate the characteristics and the techniques of the Nielsen fixed point theory method.

Most of this research was done while I was a guest of the Istituto Matematico "Ulisse Dini" of the University of Florence and of the Mathematics Research Centre of the University of Warwick. I thank especially Professors Massimo Furi and Pierluigi Zezza for their help and encouragement.

1. Fixed point theory. Let $F: X \rightarrow X$ be a map (that is, a continuous function) of a topological space. Suppose that for some subset $S$ of $X$ and for $W$ containing $S \cup F(S)$ there exists a retraction $\rho: W \rightarrow S$, that is, a map such that $\rho \mid S$, the restriction of $\rho$ to $S$, is the identity. Then the retract $f: S \rightarrow S$ of the map $F$ with respect to $\rho$ is defined by $f=\rho(F \mid S)$. The map $F$ is retractable onto $S$ with respect to $\rho$ if $\operatorname{Fix}(f)=\operatorname{Fix}(F) \cap S$, where Fix( ) denotes the set of fixed points. (For a more leisurely exposition of these definitions, see the first three sections of [1].) It will be convenient to have the following alternative definitions of retractability which are easily shown to be equivalent:

Proposition 1.1. Let $F: X \rightarrow X$ be a map, then the following are equivalent:

(a) $F$ is retractable onto $S$ with respect to $\rho: W \rightarrow S$

(b) if $y \in W \backslash S$ and $\rho(y)=x$, then $y \neq F(x)$

(c) if $x \in S$ such that $\rho F(x)=x$, then $F(x)=x$.

A subset of a space is called precompact if its closure is compact. Now suppose that $X$ is a normed linear space and that $F: X \rightarrow X$ is completely continuous, that is, $F$ is continuous and, for any bounded subset $B$ of $X$, the set $F(B)$ is precompact. Thus if such an $F$ is retractable onto a bounded set $S$, then its retract $f$ is a compact map, that is, its image $f(S)$ is precompact. If, moreover, $S$ is an absolute neighborhood retract (ANR), then there is a fixed point index theory that applies to $f$ [5] and therefore the Nielsen number $N(f)$ can be defined as in [12]. Maps $f, g: S \rightarrow S$ are said to be compactly homotopic if there is a compact map $\mathscr{H}: S \times[0,1] \rightarrow S$ such that $\mathscr{H}(x, 0)=f(x)$ and $\mathscr{H}(x, 1)=g(x)$ for 
all $x \in S$. The Nielsen number is a compact homotopy invariant, that is, if $f$ and $g$ are compactly homotopic maps on an ANR, then $N(f)=N(g)$. The other property of the Nielsen number we will need in the next section is:

Proposition 1.2. Let $X$ be a normed linear space, let $S$ be an ANR imbedded as a bounded subset of $X$, and let $F: X \rightarrow X$ be a completely continuous map retractable onto $S$ with retract $f: S \rightarrow S$, then $F$ has at least $N(f)$ fixed points in $S$.

In order to make use of the retractability concept in the analytic problems below, we will need to employ a stronger hypothesis based on form (b) of Proposition 1.1. For $\mu>0$ and $Q$ a subset of a normed linear space $X$, with norm denoted by \|\| , let

$$
\mathcal{N}_{\mu}(Q)=\{x \in X:\|x-q\|<\mu \text { for some } q \in Q\} .
$$

A map $F: X \rightarrow X$ will be called $\mu$-retractable onto a subset $S$ of $X$ if there exists $W$ containing $S \cup \mathscr{N}_{\mu}(F(S))$ and a retraction $\rho: W \rightarrow S$ such that if $y \in W \backslash S$ and $\rho(y)=x$ then $\|y-F(x)\|>\mu$. The relationship between retractability and $\mu$-retractability we will exploit is described by:

Proposition 1.3. If $U, V: X \rightarrow X$ are maps on a normed linear space such that, for some $\mu>0$, the map $U$ is $\mu$-retractable onto a subset $S$ with respect to a retraction $\rho$ and $\|V(x)\|<\mu$ for all $x \in S$, then the map $F=U+V: X \rightarrow X$ is retractable onto $S$ with respect to the same retraction.

Proof. Suppose $y=F(x)$ for some $x \in S$, then $\|y-U(x)\|=$ $\|V(x)\|<\mu$ by hypothesis. Since $U$ is $\mu$-retractable, we know $\mathscr{N}_{\mu}(U(S))$ $\subseteq W$ so $y \in W$ and we have shown that $S \cup F(S) \subseteq W$. Now if $y$ is a point of $W \backslash S$ and $x=\rho(y)$, then $y \neq F(x)$ because otherwise $\|V(x)\|$ $=\|y-U(x)\| \geq \mu$, contrary to hypothesis. Therefore $F$ is retractable with respect to $\rho$ by Proposition 1.1(b).

The examples in $\$ 5$ will make use of two easily-established results concerning $\mu$-retractability of maps $F: X \rightarrow X$, where $X=\mathbf{R}^{n}, n=1,2$.

Let $S=[r, R]$ be a closed interval where $r>0$, let $W=(0, \infty)$ and define $\rho_{r, R}: W \rightarrow S$ by

$$
\rho_{r, R}(x)= \begin{cases}r & \text { if } 0<x \leq r \\ x & \text { if } r \leq x \leq R \\ R & \text { if } x \geq R\end{cases}
$$


Proposition 1.4. Let $F: \mathbf{R}^{1} \rightarrow \mathbf{R}^{1}$ be a map and suppose there exist real numbers $0<\mu<r<R$ such that

(a) $F(x)>\mu$ for all $x$ with $r \leq x \leq R$,

(b) $F(r) \geq r+\mu$,

(c) $F(R) \leq R-\mu$.

Then $F$ is $\mu$-retractable onto $S=[r, R]$ with respect to $\rho_{r, R}:(0, \infty) \rightarrow S$.

It is convenient at this point to establish the notation we will use for vectors in $\mathbf{R}^{n}$. Let $x, y \in \mathbf{R}^{n}$ so $x=\left(x_{1}, x_{2}, \ldots, x_{n}\right)$ and similarly for $y$; then $x \cdot y=x_{1} y_{1}+x_{2} y_{2}+\cdots+x_{n} y_{n}$ and $|x|=(x \cdot x)^{1 / 2}$.

Now taking $n=2$, suppose we have real numbers $0<r<R$ and we define the annulus

$$
A_{r, R}=\left\{x \in \mathbf{R}^{2}: r \leq|x| \leq R\right\} .
$$

Letting $W=\mathbf{R}^{2} \backslash 0$, we have a retraction $\rho_{r, R}: W \rightarrow A_{r, R}$ defined by

$$
\rho_{r, R}(x)= \begin{cases}\frac{r}{|x|} x & \text { if } 0<|x| \leq r, \\ x & \text { if } r \leq|x| \leq R, \\ \frac{R}{|x|} x & \text { if }|x| \geq R .\end{cases}
$$

Proposition 1.4 generalizes to the plane in the following way:

Proposition 1.5. Let $F: \mathbf{R}^{2} \rightarrow \mathbf{R}^{2}$ be a map and suppose there exist real numbers $0<\mu<r<R$ such that

(a) $|F(x)|>\mu$ for all $x$ with $r \leq|x| \leq R$,

(b) $|x|=r$ implies $|F(x)| \geq r+\mu$,

(c) $|x|=R$ implies $|F(x)| \leq R-\mu$.

Then $F$ is $\mu$-retractable onto $S=A_{r, R}$ with respect to $\rho_{r, R}: \mathbf{R}^{2} \backslash 0 \rightarrow S$.

2. Operator theory. Let $E$ and $F$ be Banach spaces, let $L: E \rightarrow F$ be an isomorphism, let $H: E \times \mathbf{R}^{n} \rightarrow F$ be a completely continuous map, and let $B: E \rightarrow \mathbf{R}^{n}$ be a bounded linear operator onto $\mathbf{R}^{n}$. We will be concerned with the problem: find $(y, \lambda) \in E \times \mathbf{R}^{n}$ such that

$$
\left\{\begin{array}{l}
L y=H(y, \lambda) \\
B y=0
\end{array}\right.
$$

We will define a self-mapping $T$ of $E \times \mathbf{R}^{n}$ whose fixed points are the solutions of $(*)$. For that purpose, we let $E_{0}$ denote the kernel of $B$ and 
use the following easily proved fact:

LeMma 2.1. Let $\sigma: \mathbf{R}^{n} \rightarrow E$ be a linear mapping such that $B \sigma: \mathbf{R}^{n} \rightarrow \mathbf{R}^{n}$ is the identity. The map $\gamma: E \rightarrow E_{0} \times \mathbf{R}^{n}$ defined by $\gamma(x)=(x-\sigma B x, B x)$ is an isomorphism with inverse defined by $\gamma^{-1}(u, \lambda)=u+\sigma(\lambda)$.

Define $H^{+}: E \times \mathbf{R}^{n} \rightarrow F$ by $H^{+}(u, \lambda)=H(u, \lambda)+L \sigma(\lambda)$, then $T$ is the composition

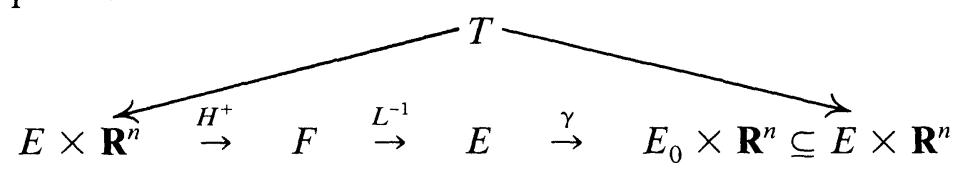

It is not difficult to show that if $H$ is a completely continuous function, then $T$ is also completely continuous.

THEOREM 2.2. The fixed points of $T$ are the solutions to $(*)$.

Proof. Suppose $T(y, \lambda)=(y, \lambda)$; then $B y=0$ because the image of $T$ is in $E_{0} \times \mathbf{R}^{n}$. Furthermore, by the definition of $T$ we are assuming that $\gamma L^{-1} H^{+}(y, \lambda)=(y, \lambda)$ which is the same as

$$
L^{-1} H^{+}(y, \lambda)=\gamma^{-1}(y, \lambda)=y+\sigma(\lambda)
$$

by the lemma. If we apply the linear function $L$ to both sides, the equation becomes $H^{+}(y, \lambda)=L y+L \sigma(\lambda)$. Since we defined $H^{+}(y, \lambda)$ $=H(y, \lambda)+L \sigma(\lambda)$, we have shown that $L y=H(y, \lambda)$ also holds. Conversely, suppose $(y, \lambda)$ satisfies $L y=H(y, \lambda)$; then from the definition we have $L^{-1} H^{+}(y, \lambda)=y+\sigma(\lambda)$. If we also suppose that $B y=0$, then $y+\sigma(\lambda)=\gamma^{-1}(y, \lambda)$ by the lemma and thus $T(y, \lambda)=(y, \lambda)$.

We wish to find conditions on the problem $(*)$ so that $T$ is retractable onto an ANR imbedded as a bounded subset of $E \times \mathbf{R}^{n}$. Then Proposition 1.2 will, in principle at least, produce multiple solutions to $(*)$. For that purpose, we introduce a condition on $H$ which we require for the rest of the paper:

Hypothesis: $H(0, \lambda) \neq 0$ for some $\lambda \in \mathbf{R}^{n}$.

Writing $T: E \times \mathbf{R}^{n} \rightarrow E \times \mathbf{R}^{n}$ in coordinate form as $T=\left(T^{(1)}, T^{(2)}\right)$, let us focus for a moment on the map $T^{(2)}: E \times \mathbf{R}^{n} \rightarrow \mathbf{R}^{n}$. From the definitions and the linearity of $B$ it follows that we can write

$$
T^{(2)}(y, \lambda)=\lambda+B L^{-1} H(y, \lambda) .
$$


For $\lambda \in \mathbf{R}^{n}$, define $H_{\lambda}, \Phi_{\lambda}: E \rightarrow F$ by $H_{\lambda}(y)=H(y, \lambda)$ and $\Phi_{\lambda}(y)=$ $H_{\lambda}(y)-H_{\lambda}(0)$. The hypothesis assures us that this is a useful definition at least to the extent that $\Phi_{\lambda} \neq H_{\lambda}$ for some $\lambda$. Then we write $T^{(2)}$ in the form

$$
T^{(2)}(y, \lambda)=\Pi(\lambda)+B L^{-1} \Phi_{\lambda}(y)
$$

where $\Pi: \mathbf{R}^{n} \rightarrow \mathbf{R}^{n}$ is defined by

$$
\Pi(\lambda)=\lambda+B L^{-1} H(0, \lambda) .
$$

As a final preliminary to our main result, we say that a map $F: X \rightarrow X$ on a normed linear space satisfies the Leray-Schauder condition (cf. [9]) for some $\nu>0$ if $\|x\|=\nu$ implies $F(x) \neq \omega x$ for all $\omega>1$. More briefly, we will write that " $F$ is $\nu-L S$ ".

The result that permits us to apply $\S 1$ to problems of the form $(*)$ is:

THEOREM 2.3. Suppose there exist positive real numbers $\mu$ and $\nu$ and an ANR $S$ a compact, locally contractible subset of $\mathbf{R}^{n}$ such that

(a) $\Pi$ is $\mu$-retractable onto $S$ with respect to a retraction $\rho_{S}: W \rightarrow S$; call its retract $\pi: S \rightarrow S$,

(b) for each $\lambda \in S$, the map $L^{-1} H_{\lambda}: E \rightarrow E$ is $\nu-L S$,

(c) $\lambda \in S$ and $\|y\| \leq \nu$ imply

$$
\|H(y, \lambda)-H(0, \lambda)\|<\frac{\mu}{\left\|B L^{-1}\right\|} .
$$

Then $(*)$ has at least $N(\pi)$ solutions $(y, \lambda)$ with $\|y\| \leq \nu$ and $\lambda \in S$.

REMARKS. Before giving the proof of the theorem, we present a brief analysis which offers some insight into why the hypotheses above might lead to the stated conclusion. It is enough to know from hypothesis (a) that $\Pi$ is retractable to deduce by Proposition 1.2 that it has fixed points $\lambda$, with $j=1,2, \ldots, N(\pi)$. Since $H_{\lambda}$ is completely continuous, we can use the Schauder Fixed Point Theorem to conclude that the $\nu-L S$ condition (b) produces a solution $y_{\lambda}$ to $L y_{\lambda}=H\left(y_{\lambda}, \lambda\right)$ for each $\lambda \in S$, where $\left\|y_{\lambda}\right\| \leq \nu$. We might then propose $\left(y_{j}, \lambda_{j}\right), j=1,2, \ldots, N(\pi)$, where $L y_{J}=H\left(y_{j}, \lambda_{l}\right)$, as $N(\pi)$ distinct solutions to $(*)$. But they are usually not solutions because $\Pi\left(\lambda_{j}\right)=\lambda$, implies only that $B L^{-1} H\left(0, \lambda_{,}\right)=0$ whereas the condition we require is that $B y_{j}=0$ or, equivalently in this case (note equation (2.1)), that $B L^{-1} H\left(y_{j}, \lambda_{J}\right)=0$. The point is that in order for hypotheses (a) and (b) really to produce the predicted number $N(\pi)$ of solutions to $(*)$, they must be related by a hypothesis such as (c). In $\S 5$, we will analyse a problem concerning a parametrized differential equation 
(Proposition 5.1) which is of type $(*)$. We will show that the problem satisfies hypotheses (a) and (b) of Theorem 2.3, yet there are no nontrivial solutions. In another example (5.2), we will consider a parametrized differential system giving rise to a problem of type $(*)$ and satisfying all the hypotheses of Theorem 2.3, so there are at least $N(\pi)$ solutions $(y, \lambda)$. In fact, $\Pi$ has exactly $N(\pi)$ fixed points in $S$ in this example. However, if $\Pi\left(\lambda_{0}\right)=\lambda_{0}$, it turns out that there is no $y \in E$ such that $\left(y, \lambda_{0}\right)$ is a solution to the system. Thus the solutions provided by Theorem 2.3 are not obtained merely by solving the finite-dimensional topological fixed point problem for $\Pi$ and then combining that solution mechanically with the solution to an analytic problem arising from an a priori estimate. As Proposition 5.2 demonstrates, there is a more subtle interplay between the topology and the analysis, identified through the use of a compact-homotopy invariant: the Nielsen number.

We prove Theorem 2.3 in two steps, of which the first is:

Lemma 2.4. Let $D=D_{\nu}=\{y \in E:\|y\| \leq \nu\}$ and define $\rho_{\nu}: E \rightarrow D$ by $\rho_{\nu}(y)=(\nu /\|y\|) y$ if $\|y\| \geq \nu$ and $\rho_{\nu}(y)=y$ otherwise. Under the hypotheses of Theorem 2.3, the map $T: E \times \mathbf{R}^{n} \rightarrow E \times \mathbf{R}^{n}$ is retractable onto $D \times S$ with respect to the retraction $\rho=\rho_{\nu} \times \rho_{S}: E \times W \rightarrow D \times S$.

Proof. Define $T_{y}^{(2)}: \mathbf{R}^{n} \rightarrow \mathbf{R}^{n}$ by $T_{y}^{(2)}(\lambda)=T^{(2)}(y, \lambda)$. We claim that the hypotheses of Theorem 2.3 imply that if $\|y\| \leq \nu$, then the mapping $T_{y}^{(2)}$ is retractable onto $S$ with respect to the retraction $\rho_{S}$. To establish this claim, we use the equation (2.2) to write $T_{y}^{(2)}=\Pi+V_{y}$ where $V_{y}(\lambda)=B L^{-1} \Phi_{\lambda}(y)$. We are assuming $\|y\| \leq \nu$ so by hypothesis (c):

$$
\left\|\Phi_{\lambda}(y)\right\|=\|H(y, \lambda)-H(y, 0)\|<\frac{\mu}{\left\|B L^{-1}\right\|} .
$$

Therefore $\left\|V_{v}(\lambda)\right\|<\mu$ and hypothesis (a) supplies the other condition for Proposition 1.3 to imply this claim. Now suppose $(y, \lambda) \in D \times S$ such that $\rho T(y, \lambda)=(y, \lambda)$, that is, both $\rho_{\nu} T^{(1)}(y, \lambda)=y$ and $\rho_{S} T^{(2)}(y, \lambda)=$ $\lambda$. Since $y \in D$, the claim above tells us, by Proposition $1.1(\mathrm{c})$, that $T^{(2)}(y, \lambda)=\lambda$. Thus by equation (2.1) we conclude that $B L^{-1} H(y, \lambda)=0$. Since it is immediate from the definition that, in general,

$$
T^{(1)}(y, \lambda)=L^{-1} H(y, \lambda)-\sigma B L^{-1} H(y, \lambda)
$$

we see that

$$
T^{(1)}(y, \lambda)=L^{-1} H(y, \lambda)=L^{-1} H_{\lambda}(y)
$$

for all $(y, \lambda) \in D \times S$ such that $T^{(2)}(y, \lambda)=\lambda$. Now $\rho_{\nu} T^{(1)}(y, \lambda)=y$ means that $T^{(1)}(y, \lambda)=\omega y$ for some $\omega \geq 1$. Suppose $\omega>1$; then since we have $L^{-1} H_{\lambda}(y)=\omega y$ for this $(y, \lambda)$, hypothesis (b) tells us that 
$\|y\|<\nu$. But then the definition of $\rho_{\nu}$ implies that $T^{(1)}(y, \lambda)=y$. Thus we have shown that $T(y, \lambda)=(y, \lambda)$ so the conclusion follows from Proposition 1.1(c).

REMARK. Hypothesis (b) of Theorem 2.3 is a well-known condition for showing that the operator equation $L y=H_{\lambda}(y)$ has a solution, by the Schauder Fixed Point Theorem. Hypothesis (b) is not a statement about $T_{\lambda}^{(1)}: E \rightarrow E$ defined by $T_{\lambda}^{(1)}(y)=T^{(1)}(y, \lambda)$, except, as we saw in the proof above, when $T^{(2)}(y, \lambda)=\lambda$ for some $y \in D$. For an arbitrary $\lambda \in S$, we have no need to demand that $T_{\lambda}^{(1)}$ satisfy any Leray-Schauder type conditions. This is an important distinction because a priori estimates implying Leray-Schauder conditions are available in the literature for the operators of the form $L^{-1} H_{\lambda}$ (see Theorem 4.5 below) but not for those of the type of $T_{\lambda}^{(1)}$.

The rest of the proof of Theorem 2.3 is carried out in the next lemma. We note that $D \times S$ is an ANR because $D$ is [2], and $S$ is [7], and the cartesian product of two ANRs is an ANR [3]. Since by Lemma 2.4 we know that $T$ is retractable onto $D \times S$, then Propositions 1.2 and 2.2 tell us there are at least $N(\tau)$ solutions to $(*)$, where $\tau: D \times S \rightarrow D \times S$ is the retract of $T$ with respect to $\rho$. Thus Theorem 2.3 is immediate from:

LEMMA 2.5. Under the hypotheses of Theorem 2.3, $N(\tau)=N(\pi)$.

$$
\begin{aligned}
& \text { Proof. Define } \mathscr{H}: D \times S \times[0,1] \rightarrow D \times S \text { by } \\
& \qquad \mathscr{H}(y, \lambda, t)=\rho\left(t T^{(1)}(y, \lambda), \Pi(\lambda)+t B L^{-1} \Phi_{\lambda}(y)\right) .
\end{aligned}
$$

The mapping $\mathscr{H}$ is well-defined because hypotheses (a) and (c) of Theorem 2.3 imply that $\Pi(\lambda)+t B L^{-1} \Phi_{\lambda}(y)$ is in $W$ (compare the proof of the previous lemma). The homotopy $\mathscr{H}$ is compact because $H$ is assumed completely continuous and we may make use of the fact that the convex hull of a precompact set is precompact. Thus $\tau$ and $0 \times \pi: D \times S$ $\rightarrow D \times S$ are compactly homotopic so $N(\tau)=N(0 \times \pi)$ by [12]. It is evident from the appropriate commutativity property of the Nielsen number [1, Theorem 5.4] that $N(0 \times \pi)=N(\pi)$.

3. Parametrized differential systems. In this section, we present several types of problems concerning systems of nonlinear differential equations that can be expressed in the form

$$
\left\{\begin{array}{l}
L y=H(y, \lambda), \\
B y=0,
\end{array}\right.
$$


where $L: E \rightarrow F$ is an isomorphism of Banach spaces, $H: E \times \mathbf{R}^{n} \rightarrow F$ is a completely continuous mapping, and $B: E \rightarrow \mathbf{R}^{n}$ is a bounded linear operator onto $\mathbf{R}^{n}$.

For a map $y:[0,1] \rightarrow \mathbf{R}^{n}$, the sup norm will be written $\|y\|_{0}$, that is,

$$
\|y\|_{0}=\sup \{|y(x)|: 0 \leq x \leq 1\} \text {. }
$$

By $C_{n}^{k}[0,1]$ we denote the space of all $C^{k}$-maps $y:[0,1] \rightarrow \mathbf{R}^{n}$ with the $C^{k}$ norm:

$$
\|y\|_{k}=\max \left\{\|y\|_{0},\left\|y^{\prime}\right\|_{0}, \ldots,\left\|y^{(k)}\right\|_{0}\right\} .
$$

Proposition 3.1. Let $h:[0,1] \times \mathbf{R}^{2 n} \rightarrow \mathbf{R}^{n}$ be a continuous function. Then the problem of finding a map $y=y(x):[0,1] \rightarrow \mathbf{R}^{n}$ and a vector $\lambda \in \mathbf{R}^{n}$ satisfying

$$
\left\{\begin{array}{l}
y^{\prime}=h(x, y, \lambda) \\
y(0)=y(1)=0
\end{array}\right.
$$

can be expressed in the form $(*)$.

Proof. Let $E$ be the subspace of $C_{n}^{1}[0,1]$ consisting of maps $y$ satisfying the initial condition $y(0)=0$, let $F=C_{n}^{0}[0,1]$ and define $L: E \rightarrow F$ by $L y=y^{\prime}$. The operator $H: E \times \mathbf{R}^{n} \rightarrow F$ is the superposition (or "Nemytskii") operator $H(y, \lambda)(x)=h(x, y(x), \lambda)$; then $H$ is completely continuous by the Ascoli-Arzela Theorem. Letting $B: E \rightarrow \mathbf{R}^{n}$ be defined by $B y=y(1)$ completes the proof.

The next type is called a "three-point boundary value problem" (compare [13]).

Proposition 3.2. Let $h:[0,1] \times \mathbf{R}^{3 n} \rightarrow \mathbf{R}^{n}$ be a continuous function. Then the problem of finding a map $y:[0,1] \rightarrow \mathbf{R}^{n}$ and vector $\lambda \in \mathbf{R}^{n}$ satisfying

$$
\left\{\begin{array}{l}
y^{\prime \prime}=h\left(x, y, y^{\prime}, \lambda\right), \\
y(0)=y(1 / 2)=y(1)=0
\end{array}\right.
$$

can be expressed in the form $(*)$.

Proof. In this case, $E$ is the subspace of $C_{n}^{2}[0,1]$ consisting of maps $y$ satisfying the Dirichlet boundary condition $y(0)=y(1)=0$, again $F=$ $C_{n}^{0}[0,1]$ and now $L y=y^{\prime \prime}$. We will later use the fact that the inverse of $L$ is defined by

$$
L^{-1} y(t)=\int_{0}^{1} G(t, s) y(s) d s
$$


where $G:[0,1] \times[0,1] \rightarrow \mathbf{R}$ is defined by

$$
G(t, s)= \begin{cases}(t-1) s & \text { if } 0 \leq s \leq t \leq 1 \\ t(s-1) & \text { if } 0 \leq t \leq s \leq 1\end{cases}
$$

We again use the superposition operator for $H$ but now we set $B y=$ $y(1 / 2)$.

The following type of problem comes from [4], where it is viewed as a control problem with finite-dimensional control space.

Proposition 3.3. Let $h:[0,1] \times \mathbf{R}^{2 n} \rightarrow \mathbf{R}^{n}$ be a map and $A \in \mathbf{R}^{n} a$ given constant vector, then the problem of finding a map $y:[0,1] \rightarrow \mathbf{R}^{n}$ and a vector $\lambda \in \mathbf{R}^{n}$ satisfying

$$
\left\{\begin{array}{l}
y^{\prime \prime}=h(x, y, \lambda) \\
y(0)=y(1)=0 \\
\int_{0}^{1} y(x) d x=A
\end{array}\right.
$$

can be expressed in the form (*).

Proof. The subspace of $C_{n}^{2}[0,1]$ we use for this case consists of maps satisfying the initial condition $y(0)=0$. We let $F$ be $C_{n}^{0}[0,1] \times \mathbf{R}^{n}$ with the product norm, that is, with $\|(y, \lambda)\|=\left(\|y\|_{0}^{2}+|\lambda|^{2}\right)^{1 / 2}$. Define $L: E$ $\rightarrow F$ by $L y=\left(y^{\prime \prime}, \int_{0}^{1} y(x) d x\right)$. Setting $H(y, \lambda)(x)=(h(x, y(x), \lambda), A)$ and $B y=y(1)$ completes the proof.

The next type of problem is based (when $n=1$ ) on a model of an adiabatic tubular chemical reactor [10], in which case we could be trying to choose a value of one of the parameters $\lambda$ of the input so that the effluent leaves the reactor at a required temperature $A$.

Proposition 3.4. Let $h:[0,1] \times \mathbf{R}^{2 n} \rightarrow \mathbf{R}^{n}$ be a map, $\beta>0$ a real constant, and $A \in \mathbf{R}^{n}$ a given vector. Then the problem of finding a map $y:[0,1] \rightarrow \mathbf{R}^{n}$ and a vector $\lambda \in \mathbf{R}^{n}$ satisfying

$$
\left\{\begin{array}{l}
\beta y^{\prime \prime}-y^{\prime}=h(x, y, \lambda), \\
\beta y^{\prime}(0)=y(0) \quad \text { and } y^{\prime}(1)=0 \text { and } y(1)=A
\end{array}\right.
$$

can be expressed in the form $(*)$. 
Proof. We still have $E \subseteq C_{n}^{2}[0,1]$ but now $y \in E$ if it satisfies the condition $\beta y^{\prime}(0)=y(0)$. Again $F=C_{n}^{0}[0,1] \times \mathbf{R}^{n}$ but here $L y=\left(\beta y^{\prime \prime}-\right.$ $\left.y^{\prime}, y(1)\right)$. The operator $H$ is exactly as in the previous proposition while we set $B y=y^{\prime}(1)$.

Let $\Omega$ be a region of $\mathbf{R}^{m}$ whose boundary $\partial \Omega$ is a smooth, compact $(m-1)$-manifold. Let $H_{n}^{2}$ be the Sobolev space of $L^{2}$-functions $y: \bar{\Omega} \rightarrow$ $\mathbf{R}^{n}$ with $L^{2}$ first and second derivatives and let $E$ be the subspace of $H_{n}^{2}$ consisting of functions that are zero on $\partial \Omega$. The Laplacian $\Delta: E \rightarrow L^{2}(\Omega)$ $=F$ is an isomorphism. Define $B: E \rightarrow \mathbf{R}^{n}$ by $B y=\int_{\Omega} y(x) d x$. Making use of the superposition operator as before, we have

Proposition 3.5. Let $h: \bar{\Omega} \times \mathbf{R}^{(m+2) n} \rightarrow \mathbf{R}^{n}$ be a map. Then the problem of finding an $L^{2}$-function $y=\left(y_{1}, y_{2}, \ldots, y_{n}\right): \bar{\Omega} \rightarrow \mathbf{R}^{n}$ and a vector $\lambda \in \mathbf{R}^{n}$ satisfying

$$
\left\{\begin{array}{l}
\Delta y=h\left(x, y, \partial y_{1} / \partial x_{1}, \ldots, \partial y_{n} / \partial x_{m}, \lambda\right) \\
y \mid \partial \Omega=0 \\
\int_{\Omega} y(x) d x=0
\end{array}\right.
$$

can be expressed in the form $(*)$.

4. Three-point boundary value problems. We will discuss problems of the form

$$
\left\{\begin{array}{l}
y^{\prime \prime}=h\left(x, y, y^{\prime}, \lambda\right), \\
y(0)=y(1 / 2)=y(1)=0,
\end{array}\right.
$$

where $h:[0,1] \times \mathbf{R}^{3 n} \rightarrow \mathbf{R}^{n}$ is a map, as in Proposition 3.2. The main result of this section, Corollary 4.6, presents sufficient conditions on $h$ so that hypothesis (b) of Theorem 2.3 is satisfied by these three-point boundary value problems. We will make use of this result in the final section. In addition, the result on which it is based (Theorem 4.5) illustrates how estimates in the literature may be modified to verify hypothesis (b).

From the proof of Proposition 3.2, we observe that

LeMma 4.1. The map $\Pi: \mathbf{R}^{n} \rightarrow \mathbf{R}^{n}$ is defined by

$$
\Pi(\lambda)=\lambda+\int_{0}^{1} g(s) h(s, 0,0, \lambda) d s,
$$


where

$$
g(s)= \begin{cases}-\frac{s}{2} & \text { if } 0 \leq s \leq 1 / 2 \\ \frac{s-1}{2} & \text { if } 1 / 2 \leq s \leq 1\end{cases}
$$

and also that

LEMMA 4.2. For the bounded linear operator $B L^{-1}: F \rightarrow \mathbf{R}^{n}$ we have $\left\|B L^{-1}\right\|<(48)^{-1 / 2}$.

Proof. Let $u=\left(u_{1}, u_{2}, \ldots, u_{n}\right):[0,1] \rightarrow \mathbf{R}^{n}$ be an element of $F$ with $\|u\|_{0} \leq 1$. Then we use Schwarz's Inequality to see that

$$
\begin{aligned}
\left|B L^{-1} u\right|^{2} & =\sum_{j=1}^{n}\left(\int_{0}^{1} g(s) u_{j}(s) d s\right)^{2} \leq \sum_{j=1}^{n}\left(\int_{0}^{1}|g(s)|\left|u_{j}(s)\right| d s\right)^{2} \\
& \leq \int_{0}^{1}|g(s)|^{2} d s \int_{0}^{1}|u(s)|^{2} d s<\int_{0}^{1}|g(s)|^{2} d s=\frac{1}{48} .
\end{aligned}
$$

The next two results are preliminaries to the theorem that follows them. The first is an elementary exercise:

LEMMA 4.3. If $c_{1}, c_{2}$ are non-negative constants, not both zero, and $0 \leq \sigma<1$, then the function $f: \mathbf{R} \rightarrow \mathbf{R}$ defined by $f(x)=x-c_{1}-c_{2} x^{\sigma}$ has exactly one positive zero.

For a map $y:[0,1] \rightarrow \mathbf{R}^{n}$ and any $p \geq 1$, the $L^{p}$ norm is

$$
\|y\|_{(p)}=\left(\int_{0}^{1}|y(x)|^{p} d x\right)^{1 / p}
$$

Note how the notation distinguishes between the $C^{2}$ norm $\|y\|_{2}$ and the $L^{2}$ norm $\|y\|_{(2)}$.

LEMMA 4.4. If $0 \leq \sigma<1$, then

$$
\int_{0}^{1}|y(x)|^{1+\sigma} d x \leq\|y\|_{(2)}^{1+\sigma} \text {. }
$$

Proof. First suppose that $\sigma \geq 1 / 2$, then by Schwarz's Inequality

$$
\begin{aligned}
\int_{0}^{1}|y(x)|^{1+\sigma} d x & =\int_{0}^{1}|y(x)||y(x)|^{\sigma} d x \\
& \leq\left(\int_{0}^{1}|y(x)|^{2} d x\right)^{1 / 2}\left(\left[\int_{0}^{1}|y(x)|^{2 \sigma} d x\right]^{1 / 2 \sigma}\right)^{\sigma} \\
& =\|y\|_{(2)}\|y\|_{(2 \sigma)}^{\sigma}<\|y\|_{(2)}^{1+\sigma}
\end{aligned}
$$


where the last inequality depends on Hölder's Inequality because we then have that $1 \leq p<p^{\prime}$ implies $\|y\|_{(p)}<\|y\|_{\left(p^{\prime}\right)}$. Now suppose that $\sigma<1 / 2$. Then similarly,

$$
\begin{aligned}
\int_{0}^{1}|y(x)|^{1+\sigma} d x & =\int_{0}^{1}|y(x)|^{1 / 2}|y(x)|^{1 / 2+\sigma} d x \\
& <\|y\|_{(1)}^{1 / 2}\|y\|_{(1+2 \sigma)}^{1 / 2+\sigma}<\|y\|_{(2)}^{1+\sigma} .
\end{aligned}
$$

Now we are ready to establish an a priori estimate for solutions to a class of Dirichlet boundary value problems for ordinary differential systems. The argument follows one in [6], but we give it in some detail because we need an explicit description of the a priori bound whereas all that was required for [6] was a proof that some bound exists. In order to obtain an easily-stated result, we considerably strengthen the hypotheses of the original result, Theorem 8.1, in particular limiting ourselves to second-order systems in which no first-derivative terms appear.

THeOREM 4.5. Suppose $h:[0,1] \times \mathbf{R}^{n} \rightarrow \mathbf{R}^{n}$ is a map for which there exist non-negative constants $c_{1}, c_{2}$, not both zero, and $0 \leq \sigma<1$ such that

$$
|h(x, u)| \leq c_{1}+c_{2}|u|^{\sigma}
$$

for all $x \in[0,1]$ and $u \in \mathbf{R}^{n}$, then a solution $y \in C_{n}^{2}[0,1]$ to

$$
\left\{\begin{array}{l}
y^{\prime \prime}=h(x, y) \\
y(0)=y(1)=0
\end{array}\right.
$$

has the property $\|y\|_{2}<3 \zeta$, where $\zeta$ is the positive zero of $f(x)=x-c_{1}-$ $c_{2} x^{\sigma}$.

Proof. Integration by parts and the boundary conditions imply

$$
\int_{0}^{1} y(x) y^{\prime \prime}(x) d x=-\int_{0}^{1}\left|y^{\prime}(x)\right|^{2} d x .
$$

Thus, using Lemma 4.4,

$$
\begin{aligned}
\left\|y^{\prime}\right\|_{(2)}^{2} & \leq \int_{0}^{1}|y(x)|\left|y^{\prime \prime}(x)\right| d x \leq \int_{0}^{1}|y(x)|\left[c_{1}+c_{2}|y(x)|^{\sigma}\right] d x \\
& \leq c_{1}\|y\|_{(2)}+c_{2} \int_{0}^{1}|y(x)|^{1+\sigma} d x<c_{1}\|y\|_{(2)}+c_{2}\|y\|_{(2)}^{1+\sigma} \\
& \leq c_{1}\left\|y^{\prime}\right\|_{(2)}+c_{2}\left\|y^{\prime}\right\|_{(2)}^{1+\sigma} .
\end{aligned}
$$


The argument for the last inequality is that $\|y\|_{(2)} \leq\|y\|_{0}$ and the condition $y(0)=0$, the Fundamental Theorem of Calculus, and Schwarz's Inequality imply that $|y(x)| \leq\left\|y^{\prime}\right\|_{(2)}$ for all $x$.

We have shown that $\left\|y^{\prime}\right\|_{(2)}$ must satisfy the inequality

$$
\left\|y^{\prime}\right\|_{(2)}<c_{1}+c_{2}\left\|y^{\prime}\right\|_{(2)}^{\sigma} \text {. }
$$

The function $f(x)=x-c_{1}-c_{2} x^{\sigma}$ obviously has the property, for $x>0$, that $f(x)<0$ only if $x<\zeta$, so $\left\|y^{\prime}\right\|_{(2)}<\zeta$. Thus $\|y\|_{0}<\zeta$ also.

From the differential equation,

$$
\left|y^{\prime \prime}(x)\right|<c_{1}+c_{2}|y(x)|^{\sigma}<c_{1}+c_{2} \zeta^{\sigma}=\zeta
$$

and therefore $\left\|y^{\prime \prime}\right\|_{0}<\zeta$.

Now

$$
\begin{aligned}
\left|y^{\prime}(0)\right| & \leq\left|y^{\prime}(x)\right|+\int_{0}^{x}\left|y^{\prime \prime}(s)\right| d s \\
& \leq\left|y^{\prime}(x)\right|+\left\|y^{\prime \prime}\right\|_{0}<\left|y^{\prime}(x)\right|+\zeta,
\end{aligned}
$$

so

$$
\left|y^{\prime}(0)\right|-\zeta<\left\|y^{\prime \prime}\right\|_{(2)}<\zeta
$$

and

$$
\left|y^{\prime}(x)\right| \leq\left|y^{\prime}(0)\right|+\left|\int_{0}^{x} y^{\prime \prime}(s) d s\right|<3 \zeta .
$$

Thus $\left\|y^{\prime}\right\|_{0}<3 \zeta$ so $\|y\|_{2}<3 \zeta$ also.

Corollary 4.6. Suppose that for some subset $S$ of $\mathbf{R}^{n}$ there exist non-negative constants $c_{1}, c_{2}$, not both zero, and $0 \leq \sigma<1$ such that

$$
|h(x, y, \lambda)|<c_{1}+c_{2}|y|^{\sigma}
$$

for all $0 \leq x \leq 1, y \in \mathbf{R}^{n}$, and $\lambda \in S$, then $L^{-1} H_{\lambda}: E \rightarrow E$ is $3 \zeta-L S$, where $\zeta$ is the positive zero of $f(x)=x-c_{1}-c_{2} x^{\sigma}$.

Proof. Suppose $y \in E$ such that $L^{-1} H_{\lambda}(y)=\omega y$ for some $\omega>1$ and some $\lambda \in S$. Then $y(0)=y(1)=0$ by the definition of $E$ in Proposition 3.2 , and also $\omega y^{\prime \prime}(x)=h(x, y, \lambda)$. Thus $y$ is a solution to

$$
\left\{\begin{array}{l}
y^{\prime \prime}=\frac{1}{\omega} h(x, y, \lambda), \\
y(0)=y(1)=0
\end{array}\right.
$$

which certainly satisfies the hypotheses of Theorem 4.5 , so $\|y\|_{2}<3 \zeta$. 
5. Examples. This first example was constructed with substantial help from Tim Poston and David Sanchez.

\section{PROPOSITION 5.1. The problem}

$$
\left\{\begin{array}{l}
y^{\prime}=K y^{1 / 3}-\lambda e^{-\lambda / 100} x+\lambda^{1 / 3} \\
y(0)=y(1)=0
\end{array}\right.
$$

satisfies hypothesis (a) of Theorem 2.3 for an interval $S=[r, R] \subseteq \mathbf{R}, r>0$, independent of the choice of the positive constant $K$, with $N(\pi)=1$, and it satisfies hypothesis (b), but for $K \geq 60$, it has no nontrivial solutions. However, if $K \leq 1 / 4$, then there is at least one solution $(y, \lambda)$ with $\lambda \in S$.

Proof. To verify hypothesis (a) of Theorem 2.3, we calculate (note Proposition 3.1) that

$$
\Pi(\lambda)=\lambda+\int_{0}^{1}\left(-\lambda e^{-\lambda / 100} s+\lambda^{1 / 3}\right) d s=\lambda\left(1-\frac{1}{2} e^{-\lambda / 100}\right)+\lambda^{1 / 3}
$$

Using Proposition 1.4, we find that $\Pi$ is $1 / 2$-retractable onto $S=$ $[1 / 2,9 / 2]$ and from the definition of the Nielsen number [8] we see that $N(\pi)=1$. We emphasize that neither the choice of $S$ nor the value of $N(\pi)$ depended on $K$.

Next we wish to verify the hypothesis (b). If $\lambda \in S$ and $y$ is a solution to the initial value problem

$$
y^{\prime}=K y^{1 / 3}-\lambda e^{-\lambda / 100} x+\lambda^{1 / 3}, \quad y(0)=0,
$$

then

$$
\begin{aligned}
\left|y^{\prime}(x)\right| & =\left|K(y(x))^{1 / 3}-\lambda e^{-\lambda / 100} x+\lambda^{1 / 3}\right| \\
& \leq K|(y(x))|^{1 / 3}+\left|-\lambda e^{-\lambda / 100} x+\lambda^{1 / 3}\right| \leq K\left\|y^{\prime}\right\|_{0}^{1 / 3}+2.65
\end{aligned}
$$

so

$$
\left\|y^{\prime}\right\|_{0} \leq K\left\|y^{\prime}\right\|_{0}^{1 / 3}+2.65
$$

It follows that $\|y\|_{1}=\left\|y^{\prime}\right\|_{0}<(K+2.65)^{3 / 2}$. Then a proof similar to that of Corollary 4.6 shows that $L^{-1} H_{\lambda}$ is $\nu-L S$ with $\nu=(K+2.65)^{3 / 2}$.

An elementary argument establishes the fact that if $y$ is a solution to the initial value problem above, with $K \geq 60$, then $y$ is an increasing function on $[0,1]$, except, of course, when $\lambda=0$.

To show that nontrivial solutions to (*) do exist if $K$ is small, we need only verify hypothesis (c) of Theorem 2.3. For $y \in E$ with $\|y\|_{1} \leq \nu=$ $(K+2.65)^{3 / 2}$ we have (for $\lambda \in S$ )

$$
\begin{aligned}
& |H(y, \lambda)(x)-H(0, \lambda)(x)| \\
& \quad=K|y(x)|^{1 / 3} \leq K\|y\|_{1}^{1 / 3}<K(K+2.65)^{1 / 2}
\end{aligned}
$$


On the other hand, certainly $\left\|B L^{-1}\right\|=1$ for this problem, so we require

$$
K(K+2.65)^{1 / 2}<\mu=1 / 2
$$

which holds if $K \leq 1 / 4$.

Proposition 5.2. The problem

(*)

$$
\left\{\begin{array}{l}
u^{\prime \prime}=\frac{\beta^{1 / 3}}{9} v^{1 / 3}+\alpha\left[2 x^{1 / 3}-4\left(\alpha^{2}+\beta^{2}\right)^{-1 / 3}\right] \\
v^{\prime \prime}=\frac{\alpha^{1 / 3}}{9} u^{1 / 3}+\beta\left[16-2 x^{1 / 3}+4\left(\alpha^{2}+\beta^{2}\right)^{-1 / 3}\right] \\
u(0)=u(1 / 2)=u(1)=0 \\
v(0)=v(1 / 2)=v(1)=0
\end{array}\right.
$$

has at least two nontrivial solutions $(y, \lambda)=((u, v),(\alpha, \beta)) \in D \times S$. If $(y, \lambda)$ is a nontrivial solution to $(*)$, then $\Pi(\lambda) \neq \lambda$.

Proof. Using Lemma 4.1 and computing, we find that

$$
\begin{aligned}
\Pi(\lambda) & =\Pi(\alpha, \beta) \\
& =\left(\alpha+2 \eta \alpha+\frac{\alpha}{2}\left(\alpha^{2}+\beta^{2}\right)^{-1 / 3},-\beta-2 \eta \beta-\frac{\beta}{2}\left(\alpha^{2}+\beta^{2}\right)^{-1 / 3}\right)
\end{aligned}
$$

where

$$
\eta=\int_{0}^{1} s^{1 / 3} g(s) d x=\frac{9}{56}\left(2^{-4 / 3}-1\right) \quad(\text { approximately }-0.1) .
$$

We may write

$$
\begin{aligned}
\Pi(\lambda) & =\Pi(\alpha, \beta)=\left((1+2 \eta)+\frac{1}{2}\left(\alpha^{2}+\beta^{2}\right)^{-1 / 3}\right)(\alpha,-\beta) \\
& =\left((1+2 \eta)+\frac{1}{2}|\lambda|^{-2 / 3}\right)(\alpha,-\beta)
\end{aligned}
$$

so

$$
|\Pi(\lambda)|=\left((1+2 \eta)+\frac{1}{2}|\lambda|^{-2 / 3}\right)|(\alpha,-\beta)|=(1+2 \eta)|\lambda|+\frac{1}{2}|\lambda|^{1 / 3} .
$$

Let us consider the function $\phi: \mathbf{R}^{+} \rightarrow \mathbf{R}^{+}=(0, \infty)$ defined by $\phi(x)=$ $(1+2 \eta) x+\frac{1}{2} x^{1 / 3}$. We observe that $\phi(x)>x$ if $x<x_{0}$ and $\phi(x)<x$ if $x>x_{0}$, where $x_{0}=(-4 \eta)^{-3 / 2}$. Furthermore, $\phi(x)-x$ has a maximum on $\left(0, x_{0}\right)$ at $r=(-12 \eta)^{-3 / 2}$ (approximately 0.76 ) with the maximum value $\mu=(-108 \eta)^{-1 / 2}$ (approximately 0.3 ). We choose $R$ so that $R-$ $\phi(R) \geq \mu$; the value $R=6.1$ will serve the purpose. Noting that $\phi$ is an increasing function, we see that $\phi(x)>\mu$ if $x \geq r$. Since $|\Pi(\lambda)|=\phi(|\lambda|)$, 
Proposition 1.5 tells us that $\Pi$ is $\mu$-retractable onto the annulus

$$
S=A_{r, R}=\left\{\lambda \in \mathbf{R}^{2}: r \leq|\lambda| \leq R\right\}
$$

with respect to the radial retraction $\rho_{r, R}: \mathbf{R}^{2} \backslash 0 \rightarrow A_{r, R}$. Since the retraction $\rho_{r . R}$ is homotopic in $\mathbf{R}^{2} \backslash 0$ to the identity map, the retract $\pi: S \rightarrow S$ of $\Pi$ is homotopic in $\mathbf{R}^{2} \backslash 0$ to $\Pi \mid S: S \rightarrow \mathbf{R}^{2} \backslash 0$, the restriction of $\Pi$. The homotopy $\mathscr{H}: S \times[0,1] \rightarrow \mathbf{R}^{2} \backslash 0$ defined by

$$
\mathscr{H}(\lambda, t)=\mathscr{H}((\alpha, \beta), t)=\left(1+t\left(2 \eta+\frac{1}{2}|\lambda|^{-2 / 3}\right)\right)(\alpha,-\beta)
$$

shows that $\Pi \mid S$ is homotopic to reflection of $S$ about the horizontal axis, which is a map of degree -1 . Thus $\pi$ is a map of degree -1 . On the other hand, $N(\pi)=\mid($ degree of $\pi)-1 \mid$ by [8], so $N(\pi)=2$. We note for later use that $\operatorname{Fix}(\pi)=\operatorname{Fix}(\Pi) \cap S$ (since $\Pi$ is retractable onto $S$ ), which in this particular case consists of precisely two points: $\left((-4 \eta)^{-3 / 2}, 0\right)$ and $\left(-(-4 \eta)^{-3 / 2}, 0\right)$.

Now we wish to use Corollary 4.6 to verify hypothesis (b) of Theorem 2.3. We write

$$
\begin{aligned}
h(x, y, \lambda)= & \left(\frac{\beta^{1 / 3}}{9} v(x)^{1 / 3}+\alpha\left[2 x^{1 / 3}-4\left(\alpha^{2}+\beta^{2}\right)^{-1 / 3}\right]\right. \\
& \left.\frac{\alpha^{1 / 3}}{9} u(x)^{1 / 3}+\beta\left[16-2 x^{1 / 3}+4\left(\alpha^{2}+\beta^{2}\right)^{-1 / 3}\right]\right) \\
= & \left(\frac{\beta^{1 / 3}}{9} v(x)^{1 / 3}, \frac{\alpha^{1 / 3}}{9} u(x)^{1 / 3}\right) \\
& +\left(2 x^{1 / 3}-4\left(\alpha^{2}+\beta^{2}\right)^{-1 / 3}\right)(\alpha,-\beta)+(0,16 \beta)
\end{aligned}
$$

so that by the triangle inequality

$$
\begin{aligned}
|h(x, y, \lambda)| \leq & \left|\left(\frac{\beta^{1 / 3}}{9} v(x)^{1 / 3}, \frac{\alpha^{1 / 3}}{9} u(x)^{1 / 3}\right)\right| \\
& +\left.\left|2 x^{1 / 3}\right| \lambda|-4| \lambda\right|^{1 / 3}|+16| \beta \mid \\
\leq & 105+\left|\left(\frac{\beta^{1 / 3}}{9} v(x)^{1 / 3}, \frac{\alpha^{1 / 3}}{9} u(x)^{1 / 3}\right)\right| \\
\leq & 105+\frac{\sqrt{2}|\lambda|^{1 / 3}}{9}|y(x)|^{1 / 3} \leq 105+(.29)|y(x)|^{1 / 3} .
\end{aligned}
$$

Thus $L^{-1} H_{\lambda}$ is $3 \zeta-L S$ for all $\lambda \in S$ where $\zeta$ is the positive zero of $f(x)=x-105-(.29) x^{1 / 3}$. We may take $\zeta=107$. 
Turning to hypothesis (c), we note from Lemma 4.2 that

$$
\frac{\mu}{\left\|B L^{-1}\right\|} \geq \sqrt{48} \mu=\sqrt{\frac{48}{108 \eta}}>2.1 \text {. }
$$

On the other hand, for $\lambda \in S$ and $\|y\|_{2} \leq 3 \zeta$ we have $|y(x)| \leq 321$ for all $0 \leq x \leq 1$ so

$$
\begin{aligned}
|H(y, \lambda)(x)-H(0, \lambda)(x)| & =\left|\left(\frac{\beta^{1 / 3}}{9} v(x)^{1 / 3}, \frac{\alpha^{1 / 3}}{9} u(x)^{1 / 3}\right)\right| \\
& \leq(.29)(321)^{1 / 3}<2.1<\frac{\mu}{\left\|B L^{-1}\right\|} .
\end{aligned}
$$

Therefore, Theorem 2.3 tells us that the problem $(*)$ has at least two solutions $(y, \lambda)$ with $\|y\|_{2} \leq 321$ and $(-12 \eta)^{-3 / 2} \leq|\lambda| \leq 6.1$.

It remains to show that the fixed points $\lambda= \pm\left((-4 \eta)^{-3 / 2}, 0\right)$ of $\Pi$ do not themselves produce solutions to $(*)$. We will consider $\lambda=$ $\left((-4 \eta)^{-3 / 2}, 0\right)$; the other case is almost identical. Since $\beta=0$, the first differential equation of $(*)$ becomes

$$
u^{\prime \prime}(x)=2 \alpha x^{1 / 3}-4 \alpha^{1 / 3}
$$

where $\alpha=(-4 \eta)^{-3 / 2}=\left[(9 / 14)\left(1-2^{-4 / 3}\right)\right]^{-3 / 2}$. Integrating and using the boundary conditions $u(0)=u(1)=0$, we find that

$$
u(x)=(14 / 9)^{1 / 2}\left(1-2^{-4 / 3}\right)^{-3 / 2} \theta(x)
$$

where $\theta(x)=x^{7 / 3}+\left(2^{-1 / 3}-2\right) x^{2}+\left(1-2^{-1 / 3}\right) x$, which has the property $\theta(1 / 2)=u(1 / 2)=0$. Thus the second differential equation is

$$
\begin{aligned}
v^{\prime \prime}(x) & =\frac{1}{9} \alpha^{1 / 3}(u(x))^{1 / 3}=\frac{1}{9}\left(\frac{14}{9}\right)^{2 / 3}\left(1-2^{-4 / 3}\right)^{-1}(\theta(x))^{1 / 3} \\
& =M(\theta(x))^{1 / 3}
\end{aligned}
$$

where $M$ is a positive constant. So the only solution $y=(u, v)$ possible for this value of $\lambda$ must be

$$
v(x)=M \int_{0}^{1} G(x, s)(\theta(s))^{1 / 3} d s
$$

where $G$ is as in the proof of Proposition 3.2. In particular

$$
v(1 / 2)=M \int_{0}^{1} g(s)(\theta(s))^{1 / 3} d s
$$

where $g(s)$ is as in Lemma 4.1, that is

$$
\begin{aligned}
v(1 / 2) & =M\left\{\int_{0}^{1 / 2}\left(-\frac{s}{2}\right)[\theta(s)]^{1 / 3} d s+\int_{1 / 2}^{1} \frac{s-1}{2}[\theta(s)]^{1 / 3} d s\right\} \\
& =\left(-\frac{M}{2}\right) \int_{0}^{1 / 2} s\left([\theta(s)]^{1 / 3}+[\theta(1-s)]^{1 / 3}\right) d s .
\end{aligned}
$$


An elementary argument shows that $\theta(s)+\theta(1-s) \geq 0$ for $0 \leq s \leq 1 / 2$ so $v(1 / 2)<0$ and we conclude that there is no solution $(y, \lambda)$ to the three-point boundary value problem $(*)$ with $\lambda=\left((-4 \eta)^{-3 / 2}, 0\right)$.

\section{REFERENCES}

[1] R. Brown, Retraction methods in Nielsen fixed point theory, Pacific J. Math., 115 (1984), 277-297.

[2] J. Dugundji, An extension of Tietze's theorem, Pacific J. Math., 1 (1951), 353-367.

[3] , Topology, Allyn and Bacon, 1966.

[4] M. Furi, P. Nistri, M. Pera and P. Zezza, Topological methods for the global controllability of non-linear systems, J. Optim. Theory Appl., 45 (1985), 231-256.

[5] A. Granas, The Leray-Schauder index and the fixed point theory for arbitrary ANRs, Bull. Soc. Math. France, 100 (1972), 209-228.

[6] A. Granas, R. Guenther and J. Lee, Nonlinear boundary value problems for some classes of ordinary differential equations, Rocky Mountain J. Math., 10 (1980), 35-58.

[7] S. Hu, Theory of Retracts, Wayne State, 1965.

[8] B. Jiang, Lectures on Nielsen fixed point theory, Contemporary Math., Vol. 14, 1983.

[9] W. Kirk and C. Morales, Condensing mappings and the Leray-Schauder boundary condition, Nonlinear Anal., 3 (1979), 533-538.

[10] R. Leggett and L. Williams, Multiple positive fixed points of non-linear operators on ordered Banach spaces, Indiana U. Math. J., 28 (1979), 673-688.

[11] J. Leray, Le theorie des points fixes et ses applications en analyse, Proc. International Congress of Math., 1950, Vol. 2, Amer. Math. Soc., 1952.

[12] K. Scholz, The Nielsen fixed point theory for non-compact spaces, Rocky Mountain J. Math., 4 (1974), 81-87.

[13] J. Thomas and D. Zachmann, A nonlinear three-point boundary value problem, J. Math. Anal. Appl., 58 (1977), 647-652.

Received February 20, 1986.

UNIVERSITY OF CALIFORNIA, LoS ANGELES

LOS ANGELES, CA 90024 



\section{PACIFIC JOURNAL OF MATHEMATICS EDITORS}

\author{
V. S. VARADARAJAN \\ (Managing Editor) \\ University of California \\ Los Angeles, CA 90024 \\ Herbert Clemens \\ University of Utah \\ Salt Lake City, UT 84112 \\ R. FINN \\ Stanford University \\ Stanford, CA 94305
}

ROBION KIRBY

University of California

Berkeley, CA 94720

C. C. MOORE

University of California

Berkeley, CA 94720

HAROLD STARK

University of California, San Diego

La Jolla, CA 92093

\section{ASSOCIATE EDITORS}
R. ARENS
E. F. BECKENBACH
B. H. NEUMANN
F. WOLF
K. YOSHIDA (1906-1982)

\section{SUPPORTING INSTITUTIONS}
UNIVERSITY OF ARIZONA
UNIVERSITY OF OREGON
UNIVERSITY OF BRITISH COLUMBIA UNIVERSITY OF SOUTHERN CALIFORNIA
CALIFORNIA INSTITUTE OF TECHNOLOGY
UNIVERSITY OF CALIFORNIA
STANFORD UNIVERSITY
MONTANA STATE UNIVERSITY
UNIVERSITY OF HAWAII
UNIVERSITY OF NEVADA, RENO
UNIVERSITY OF TOKYO
NEW MEXICO STATE UNIVERSITY
UNIVERSITY OF UTAH
OREGON STATE UNIVERSITY
WASHINGTON STATE UNIVERSITY UNIVERSITY OF WASHINGTON 


\section{Pacific Journal of Mathematics}

\section{Vol. 131, No. $1 \quad$ November, 1988}

Tomek Bartoszynski, On covering of real line by null sets $\ldots \ldots \ldots \ldots \ldots 1$

Allen Davis Bell and Kenneth R. Goodearl, Uniform rank over differential operator rings and Poincaré-Birkhoff-Witt extensions $\ldots \ldots \ldots \ldots \ldots 13$

Brian Boe, Thomas Jones Enright and Brad Shelton, Determination of the intertwining operators for holomorphically induced representations of Hermitian symmetric pairs

Robert F. Brown, Topological identification of multiple solutions to

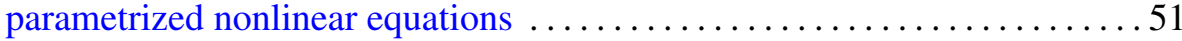

Marc R. M. Coppens, Weierstrass points with two prescribed nongaps ......71

Peter Larkin Duren and M. Schiffer, Grunsky inequalities for univalent functions with prescribed Hayman index .................... 105

Robert Greene and Hung-Hsi Wu, Lipschitz convergence of Riemannian manifolds

Kathryn E. Hare, Arithmetic properties of thin sets

Neal I. Koblitz, Primality of the number of points on an elliptic curve over a

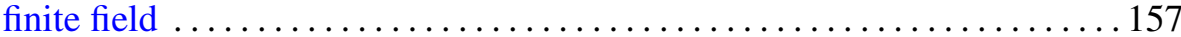

Isabel Dotti de Miatello and Roberto Jorge Miatello, Transitive isometry groups with noncompact isotropy

Raymond A Ryan, Weakly compact holomorphic mappings on Banach

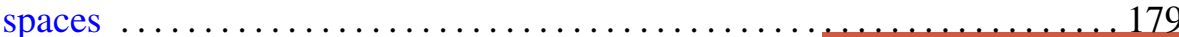

Tudor Zamfirescu, Curvature properties of typical convex surfaces 